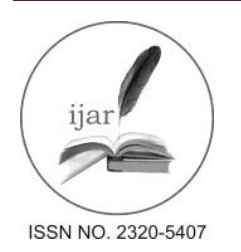

\author{
Journal homepage: http://www.journalijar.com \\ Journal DOI: $\underline{\text { 10.21474/IJAR01 }}$
}

INTERNATIONAL JOURNAL

OF ADVANCED RESEARCH

RESEARCH ARTICLE

\title{
A COMPARATIVE EVALUATION OF DENTIN WALL THICKNESS USING CONE BEAM COMPUTED TOMOGRAPHY AFTER BIO-MECHANICAL PREPARATION BY THREE DIFFERENT ROTARY FILE SYSTEMS- A CLINICAL REPORT.
}

\author{
Dr.B Rajkumar ${ }^{1}$, Dr.Anika Bhasin ${ }^{2}$, Dr.Devang Kankane², Dr.Lalit Boruah ${ }^{3}$, Dr.Vishesh Gupta ${ }^{3}$, \\ "Dr.Akanksha Bhatt". \\ 1. Professor \& Head of Department, Department of Conservative Dentistry \&Endodontics, BabuBanarasi Das \\ College of Dental Sciences, BBD University, Lucknow, india. \\ 2. Post Graduate Student, Department of Conservative Dentistry \&Endodontics, BabuBanarasi Das College of \\ Dental Sciences, BBD University, Lucknow, india. \\ 3. Associate Professor, Department of Conservative Dentistry \&Endodontics, BabuBanarasi Das College of \\ Dental Sciences, BBD University, Lucknow, india.
}

\section{Manuscript Info}

Manuscript History:

Received: 11 April 2016

Final Accepted: 13 May 2016

Published Online: June 2016

Key words:

CBCT,Dentin thickness, Hero Shaper, Hyflex CM, Root canal preparation, V-Taper..

*Corresponding Author

Dr. Akanksha Bhatt.

\section{Abstract}

Aim:- To study and compare the change in dentin wall thickness at three levels(coronal,middle and apical) for evaluating the cutting efficiency of three rotary systems.

Summary:- Root canal procedure was performed using three different rotary systems following manufacturers recommendation i.eHyflex CM (Coltene, U.S.A), V-Taper (SS White and Heroshaper (MicroMega, France), all the preparation was done uptoat $4 \%$ \#25 following standard irrigation protocol. Pre and post instrumentation dentin wall thickness was evaluated using Cone Beam Computed Tomography.

Key learning points:-

* Remaining dentin thickness is a critical factor in determining the success of post-endodontic restoration.

* All rotary files used in the present study have different cutting efficiency.

* CBCT is an important tool for evaluation of remaining dentin thickness.

Copy Right, IJAR, 2016,. All rights reserved.

\section{Introduction:-}

The objectives of biomechanical preparation are to remove the necrotic pulp tissue and micro-organisms from the root canal system followed by three- dimensional obturation. ${ }^{1}$ This bio-mechanical preparation causes inadvertent loss of some amount of coronal and radicular dentin. Preservation of remaining root canal thickness during root canal treatment is essential for strength and enhancing the longevity of an endodontically treated tooth. Studies show a direct relationship between loss of tooth structure and possibility of fracture of tooth after root canal therapy.

The design features,mechanical properties and technology used to manufacture different endodontic rotary files are different which may effect its cleaning efficiency, quantity of dentin removal and finishing of canal. ${ }^{2}$

Various studies evaluated the root canal wall thickness after instrumentation with rotary systems at different levels of the root canal ${ }^{3,4,5,6}$. The present study was designed to evaluate and compare the remaining dentin thickness after preparation with three different file systems i.eHyflexCM,V-Taper and Hero Shaper.

\section{Clinical report:-}


Threehealthy orthodontic patients were selected, slated for extraction of all first premolars and weresubjected to CBCT scan prior to instrumentation.Images were captured using i-cat CB 500 machine with total scan time of 23 seconds.

After recording data,access cavities were prepared using highspeedairotorhandpiece (NSK,PANA AIR, Japan) with endo-access bur (Dentsplymallifer, Switzerland) in rubber dam isolation.The working length was measured using iPexIIapex locator(NSK,Japan) and verified digital radiography . Bio-mechanical preparation was performed in all the four first premolars of each patient using three different file systems i.e. Hyflex in the first patient,V-Taper in secondand Hero-Shaper in third.For all the groups standard irrigation protocol was followed using $5.25 \%$ sodium hypochlorite(Pyrex), $0.9 \%$ saline(nirlife), $17 \%$ EDTA solution(Merck) and $2 \%$ chlorhexidine(V-concept)and preparation was done till $4 \%$ \#25 in all the patients.

After the preparation of canals the patient was again subjected to CBCT scans in the manner similar to Pre-scan.

The actual dentin thickness was evaluated prior and the remaining dentin thickness after instrumentation from pre and post scans respectively.

\section{Discussion:-}

The amount of dentin removed by Hyflex,V-Taper and HeroShaper were evaluated and compared using CBCT. CBCT based root canal length measurements are accurate and reliable when compared with a gold standard. ${ }^{7} \mathrm{CBCT}$ was used in this study since it can show morphological features from a three dimensional prespective, it displays axial,saggital and coronal sections. ${ }^{8}$

The Hyflex CM system was launched in 2011 by ColteneWhaledent, Switzerland. It constitutes of 6 files which has been manufactured by a unique process that controls the material's memory ${ }^{9}$, makes it extremely flexible and provides higher resistance to cyclic fatigue. These files are available with a fixed taper of 0.8, 0.6 and 0.4. Except its orifice opener i.e 0.8 taper all files have positive rake angle. The cutting profile of each file facilitates penetration in canal and presents a root canal shape corresponding with original anatomy. ${ }^{10}$ Other than this accelerated flutes design (variable pitch), no radial lands and a safe ended cutting tip is common feature in all files.It has higher resistance to cyclic fatigue and flexibility than conventional super elastic NiTi files. ${ }^{1011}$

V-TaperSS White are NiTi endodontic files with Variable taper design which creates conservative coronal shape and preserves dentin. This system is a three file system file no.17,20 and 25 which encompasses a more anatomic design that creates a shape closely mirroring the natural shape of root.

Hero shaper files (France) is in use since 2001. It is a six file system with a variable helical angle and adapted pitch. It has a positive rake angle and a blade shows a triple helix cutting edge with no radial land. ${ }^{12}$ Their helical angle increases from tip to shank that is claimed to reduce threading.

In this study, dentin thickness was measured before and after instrumentation. These values suggested that the mechanical limits of the instruments used to enlarge the root canal to approximate predetermined values would significantly weaken the dentinal walls. It has been indicated that $0.3 \mathrm{~mm}$ of canal wall should exist after canal preparation as the minimum remaining dentin thickness (RDT). This is important for providing enough resistance against lateral forces during canal filling and occlusal forces.

The result showed significant difference between Hyflex and V- Taper( p value- .002) and Hyflex and Heroshaper(p value-.029) while there were no significant differences between Heroshaper and V-Taper(p value-.223) The results of the study showed maximum cutting efficiency of Hyflex and minimum of V-Taper. Hence the amount of remaining dentin thickness which is a critical parameter for success of post-endodontic restoration is maximum in preparations done with V-taper and minimum with Hyflex. The aggressive cutting of Hyflex may be dueto highest torque and speed used during preparation than its other counterparts while standardizing the time duration for each preparation. Moreover among all these three file systems Hyflex were used with an orifice opener which allowed the consecutive files to make more contact with dentin due to coronal enlargement. Also Hyflex files have a property of controlled memory as a result of which they respond to excessive resistance with straightening of the spirals. 


\section{Conclusion:-}

The mean difference in remaining dentin thickness was highest in case of group II - V-Taper followed by group IIIHero-Shaper and least with group I-Hyflex concluding that the root canal preparation was most conservative with VTaper while Hyflex cuts more aggressively.

\section{Refrences:-}

1. HuiKhengChng, Nah Nah Chen, EngTiongKoh et al( 2004) Guidelines for root canal treatment. Singapore Dental Journal26, 60-62

2. C. Bellucci\&N.Perrini (2002) A study On the thickness of radicular dentin and cementum in anterior and premolar teeth. International Endodontic Journal 46,594-60

3. SteveMontgonery (1985) Root canal wall thickness of mandibular molars after biomechanical prepration.

4. John T. McCann, David Keller, Gary L LaBounty et al. A modification of the muffle model system to study root canal morphology.Journal of Endodontics.1990; 16: 114-1 15.

5. Charles R. Glosson, Robert H. Haller, S. Brent Dove et al. A comparison Of root canal preparations using Ni-Ti hand, Ni-Ti engine- driven and K-FIex endodontic instruments. Journal OfEndodontics. 1995; 146-151.

6. RaphalePilo, Glenda Corcino, Aviad •lamsi et al. Residual dentin thickness in mandibular premolars prepared with hand and rotary instruments, Journal Of Endodontics. 1998, 24: 401-404.

7. Y a-Hong Liang, Lan Jiang, Chen Chen et al. The validity of cone beam computed tomography in measuring root canal length xsing a gold standard. Journal OfEndodontics. 2013; 39: 1607-1610.

8. S. Patel, A. Dawood, T. Pitt Ford et al. The potential applications of Cone beam computed tomography in management of endodontic problems.International Endodontic Journal. 2007; 40: S 18-830.

9. ElizabethNinan\& David W. Berlins. Torsion and bending properties Of shape memory and super elastic Ni-Ti rotary instruments, Journal of Endodontics.2013;39:101-104

10. Peters OA, Gluskin AK, et al, An in-vitro asssesement of physical properties of novel HyflexNiTi rotary instruments. International Endodontic Journal 45,1027-34.

11. Dan Zbao, YaShen, Bin Peugct al, Micro-computed Tomography Evaluation Of the preparation Of Mesiobuccal Root Canals in Maxillary First Molars with HyFlex CM, Twisted Files, and K3 Instruments. Journal OfEndodontics. 2013, 39: 385-389.

12. G. B, Yang, X. D. Zhou, Y. L Zhang et al. Shaping ability Of progressive versus constant taper instmments in curved root canals Of extracted teeth.Intemational Endodontic Journal.2007; 40: 707-714. 